\title{
A view of brief CBT for insomnia in Japan
}

\author{
Makie Nagai* \\ Psychiatirc and Mental Health Nursing, Tsuruga Nursing University, Japan
}

${ }^{\star}$ Corresponding Author: Psychiatirc and Mental Health Nursing, Tsuruga Nursing University, Japan; Tel: +81770205514; E-mail: m-nagai@tusuruga-nu.ac.jp

Received: September 06, 2019; Accepted: September 09, 2019; Published: September 13, 2019;

Insomnia is common among older people that one out of five person complain insomnia in Japan [1]. The treatment of sleep-related illness in older patients must be undertaken with an appreciation of the physiologic changes associated with aging [2].

However one out of twenty person is using a sleeping drug in Japan [3]. The Guideline of Ministry of Health, Labor and Welfare (2013) points out to problem that multiple and massive dose drug and long-term administration. It is important issue that improvement of life style in aged person with insomnia. Furthermore epidemiologic survey showed that deficiency of sleeping time increased risk of obesity [4] and concerned with prevalence and crisis rate of hypertension [5, 6]. Insomnia is important factor of lifestyle disease and influence to aging.

Insomnia is the most frequency of sleep-related illness that is caused by chronic psychological stress and anxiety in daily life [7]. Insomnia should be treat independently (International Classification of Sleep Disorders, Third edition) and Cognitive Behavioral Therapy for Insomnia (CBT-I) is recommended as the most high evidence in non-drug treatment in US [8]. CBT-I was reported to meta-analysis of randomized controlled trial that is effectively at the end of point and the follow-up point in Japan [9]. However, it is not only CBT-I but also CBT for mental illness are not diffused in clinical fields in Japan because practitioners and time for the treatment are very few. Accordingly, Japanese nurses are expected to the practical person of $\mathrm{CBT}$ and the educational training system have been continued by Ministry of Health, Labor and Welfare. We consider that Japanese nurses should engage to CBT-I and a simple model of CBT-I is required.

Recently, brief CBT-I has been developed in UK [10] and the effectiveness was report [11]. We try to develop of brief CBT-I Japanese-version and try to the pilot study. Standard CBT-I is five or six session and take about fifty minutes, but brief CBT-I is four or three session and take about ten minutes. Our brief CBT-I focus to individual problems, and it is very specific content. If the brief CBT-I by Japanese nurses is effectively, multiple and massive dose drug among older people will improve. At the present moment, the pilot study is producing to very good results. I will engage to randomized controlled trial in the future.

\section{References}

1. Kim K, Uchiyama M, Okawa M (2000) An epidemiological study of insomnia among the Japanese general population. Sleep 23: 41-47.

2. Norman W, Osama Elkholy, Marc Baltzan, Mark Palayew (2007) Sleep and aging: 2. Management of sleep disorders in older people. CMAJ 176: 1449-1454.
3. Kaneita Y, Ohida T, Osaki Y, Tanihata T, Minowa M, et al. (2007) Association between mental health status and sleep status among adolescents in Japan: a nationwide cross-sectional survey. J Clin Psychiatry 68: 1426-1435.

4. Cappuccio FP, Taggart FM Kandala NB (2008) Gender-Specific Associations of Short Sleep Duration With Prevalent and Incident Hypertension The Whitehall II Study. Sleep 30: 619-626.

5. Gangwisch JE, Heymsfield SB, Boden-Albala B (2006) Short Sleep Duration as a Risk Factor for Hypertension Analyses of the First National Health and Nutrition Examination Survey. Hypertension 47: 833-839.

6. Gottlieb DJ, Redline S, Nieto FJ (2006) Association of Usual Sleep Duration With Hypertension: The Sleep Heart Health Sleep. Sleep 29: 1009-1014.

7. http://ghdx.healthdata.org/organizations/ministry-health-labour-and-welfare-japan.

8. Practice Parameters for the Psychological and Behavioral Treatment of Insomnia: An Update. An American Academy of Sleep Medicine Report 2006.

9. Okajima I, Komada Y, Inoue YA(2011) meta-analysis on the treatment effectiveness of cognitive behavioral therapy for primary insomnia. Sleep and Biol Rhythms 9: 24-34.

10. David L (2013) Using CBT in General Practice (2nd Edn) The 10 Minute CBT Handbook, Scion Publishing Ltd. United Kingdom.

11. Tonya M P, Sarah B E, Maggie B, et al. (2017) A single arm pilot trial of brief cognitive behavioral therapy for insomnia in adolescents with physical and psychiatric comorbidities, Jounal of Clinical Sleep Medicine 13: 401-410.
Citation:

Nagai M (2019) A view of brief CBT for insomnia in Japan. Ageing Sci Ment Health Stud Volume 3(3): 1-1. 